\title{
Lung cancer, pulmonary emphysema and pleural effusion: An autopsy study
}

\author{
Miloslav Marela, Leona Koubkovaa , Zuzana Kovarikovaa ${ }^{a}$, Alzbeta Grandcourtovaa ${ }^{a}$ Frantisek Petrika ${ }^{a}$, Hana Hroudovab, \\ Linda Capkova ${ }^{\mathrm{b}}$, Roman Kodet ${ }^{\mathrm{b}}$, Libor Fila ${ }^{\mathrm{a}}$
}

\begin{abstract}
Objectives. To determine the exact incidence of lung cancer, pulmonary emphysema and pleural effusion we decided to carry out an autopsy study.

Methods. In this autopsy study carried out over two years, we compared the results of autopsy findings with the clinical data in accompanying records of the deceased.

Results. Among the 708 deceased subjects, there were 398 males and 310 females with a median age of 71 years. At autopsy, 55 cases of lung carcinoma (BCA) were found, of which 24 have not been identified during life (44\%). Among the deceased with BCA, emphysema was also observed at autopsy in $40 \%$ of the cases. Pulmonary emphysema was described macroscopically in $28 \%$ of the full set of 708 deceased, whereas the accompanying records of the deceased described this condition in only $12 \%$ of the cases. Microscopic changes compatible with emphysema were identified in $54 \%$ of the examined lungs. Pleural effusions were described in the accompanying records of $13 \%$ of the deceased, while the autopsies showed this condition in $33 \%$ of the deceased. BCA was accompanied by effusion in $25 \%$ of the cases. Conclusions. The obtained results show that the studied conditions are present in more cases than are reported by clinicians. The study confirms the commonly accepted association between lung cancer and emphysema.
\end{abstract}

Key words: autopsy pathology, lung cancer, emphysema, pleural effusion

Received: December 10, 2014; Accepted: May 5, 2015; Available online: May 19, 2015

http://dx.doi.org/10.5507/bp.2015.024

${ }^{a}$ Department of Pneumology, $2^{\text {nd }}$ Faculty of Medicine, Charles University in Prague and Faculty Hospital Motol, Prague, Czech Republic ${ }^{b}$ Department of Pathology, $2^{\text {nd }}$ Faculty of Medicine, Charles University in Prague and Faculty Hospital Motol, Prague Corresponding author: Libor Fila, e-mail: libor.fila@fnmotol.cz

\section{INTRODUCTION}

The incidence of lung cancer (BCA) in the Czech Republic remains high, having slowly decreased in men to $90 / 100,000$ and increased in women to $35 / 100,000$ in the past thirty years ${ }^{1}$. The TNM stages I and II are diagnosed in fewer than $20 \%$ of all patients. The difference between the numbers of patients diagnosed with BCA during life and those whose tumors are identified only at autopsy is not precisely known. Likewise, we do not know the exact causes of death in patients with BCA for either those diagnosed during life or those identified at autopsy.

For many years, a stable $7.7 \%$ of the population in our country has reportedly suffered from COPD (emphysema and chronic bronchitis with obstruction) (ref. ${ }^{2}$ ).

There is a need for improved accuracy regarding the estimates of the prevalence of emphysema in the deceased as a function of age.

Many studies have shown that patients with COPD have an increased risk of developing lung cancer ${ }^{3}$. However, the prevalence of the coexistence of emphysema and BCA is in our population is also not precisely known. This knowledge (development of BCA in COPD vs. BCA in patients without COPD) may permit earlier diagnosis and allow for more effective BCA screening programs.

Epidemiological studies on pleural effusion show that it affects 0.32 to $0.5 \%$ of the population annually ${ }^{4}$.
However, knowledge regarding the relative prevalence of this condition during life as well as that diagnosed postmortem may also help to improve the diagnosis of this complication of many diseases.

To answer these questions and to find additional connections, we decided to organize an autopsy study.

\section{MATERIALS AND METHODS}

This study was designed as a prospective autopsy study including both macroscopic and microscopic observations. We compared the clinical data referenced in accompanying reports for the deceased with autopsy findings in both the adult and pediatric departments of the largest hospital in the Czech Republic, the Faculty Hospital Motol. In addition to the macroscopic description of the autopsy findings by a pathologist, the lungs were also examined microscopically by the seventh author of this article. During two years, from March $1^{\text {st }} 2011$ to February $28^{\text {th }} 2013$, we obtained a complete set of data on 708 subjects. The results of the microscopic examination of more than 600 lungs of the deceased out of the full set of 708 subjects were made available. The statistical significance of the differences in the number of cases of emphysema detected at autopsy in subjects younger and older than 60 years and the differences in the incidence 
Table 1. Lung cancer diagnosed during life and at autopsy and its association with pulmonary emphysema.

\begin{tabular}{lcc}
\hline BCA & Number of cases (\%) & Number of cases with coincident emphysema (\%) \\
\hline All cases of BCA & $55(100 \%)$ & $22(40 \%)$ \\
BCA known during life & $31(56 \%)$ & $10(32 \%)$ \\
BCA identified at autopsy & $24(44 \%)$ & $12(50 \%)$ \\
\hline
\end{tabular}

BCA: lung cancer

of lung cancer in those with and without emphysema were assessed using a chi-square test.

To test the hypothesis that the relative frequency of occurrence of emphysema does not change as a function of age, we used Pearson's chi-square test of contingency tables. The statistical evaluations were performed using the Statistica program by the StatSoft company, the Czech Republic.

\section{RESULTS}

The study group included 708 autopsied deceased subjects (398 males and 310 females) with a median age of 71 years (range $8-101$ years).

\section{Lung cancer}

At autopsy, we found a total of 55 cases of lung cancer, representing $8 \%$ of all the deceased. The group included 19 females and 36 males. The ratio was one identified BCA for every 13 autopsies.

Of these, 31 cases ( 12 females, 19 males) of BCA were diagnosed during life and another 24 cases (17 males, 7 females, $44 \%$ of all BCA) were identified only at autopsy. For the BCA at TNM stages I and II, $42 \%$ and $20 \%$ of cases were detected only at autopsy and during life, respectively. The diseases at TNM stages III and IV were identified in $58 \%$ and $80 \%$ of cases at autopsy and during life, respectively. Of the subjects with pulmonary malignancy, 22 cases (40\%) also had macroscopically described emphysema.

In the 31 patients with BCA diagnosed during life, emphysema was detected at autopsy in 10 cases (32\%). Of the 24 subjects with lung cancer detected only post-mortem, emphysema was found during autopsy in 12 cases (50\%). See Table 1.

Our results show that one newly identified BCA was observed for every 30 autopsies, i.e. that in 3\% of autopsies of the deceased in our set, there was one previously unknown BCA. The causes of death according to the autopsy reports of subjects with lung cancer identified during life (31) and diagnosed only at autopsy (24) are shown in Table 2.

Out of the total of 55 cases of BCA proven at autopsy, $14(25 \%)$ were accompanied by a pleural effusion. The effusion was most frequently bilateral ( 9 cases), but in 4 cases it was observed only on the right side and in one case only on the left side.
Table 2. Causes of death in patients with lung cancer.

\begin{tabular}{lcc}
\hline Causes of death & $\begin{array}{c}\text { BCA known } \\
\text { during life }\end{array}$ & $\begin{array}{c}\text { BCA identified } \\
\text { at autopsy }\end{array}$ \\
\hline Respiratory failure & 6 & 4 \\
Pneumonia & 5 & 4 \\
Pulmonary embolism & 3 & 2 \\
Gastrointestinal bleeding & 1 & 1 \\
Metastatic BCA & 10 & \\
Acute myocardial infarction & 2 & \\
CNS metastases & 1 & \\
Ileus & 1 & \\
Sepsis & 1 & \\
Tracheal stenosis & 1 & \\
Heart failure & & 2 \\
COPD exacerbation & & 2 \\
Stroke & & 2 \\
Other tumors & & 24 \\
Complications of DM & & \\
Total & 31 & \\
\hline
\end{tabular}

BCA: lung cancer; COPD: chronic obstructive pulmonary disease; DM: diabetes mellitus

\section{Pulmonary emphysema}

According to data from the accompanying reports on the deceased, pulmonary emphysema was diagnosed during life in 83 patients (12\%), 40 males and 43 females. Macroscopic emphysema described only at autopsy by the pathologist was found in another 112 deceased (67 males and 45 females), for a total of 195 deceased with emphysema, $28 \%$ of the entire studied group (107 males and 88 females). Thus, the prevalence of macroscopically diagnosed emphysema at autopsy was $28 \%$.

Age was found to be a factor that significantly influenced the presence of macroscopically proven emphysema found by autopsy. A total number of 135 subjects died before 59 years of age (median age, 48 years). According to the autopsy results, 12 of them (9\%) suffered from emphysema ( 6 males and 6 females). At autopsy, emphysema was recognised in only 7 cases in this group, and during life it was diagnosed in 5 of the deceased. A total of 573 of the deceased were older than 60 years of age, with a median age of 76 years. At autopsy, emphysema was reported in 183 cases (101 males and 82 females). In 105 cased, the emphysema was identified only at autopsy and in 78 subjects the diagnosis was known during life. The presence of emphysema in subjects over 60 years of age was statistically significantly higher than in younger group, 
$P<0.001$. The prevalence of emphysema macroscopically proven by autopsy in this group was $32 \%$.

\section{Lung cancer and pulmonary emphysema}

In the group of 195 subjects with macroscopic emphysema proven at autopsy we also identified 22 subjects with BCA. Thus, one case of confirmed BCA was observed per 9 subjects with emphysema (22/195). The remaining 33 cases of BCA were observed in the 513 deceased who did not have emphysema. In the emphysema-free group, there was one case of BCA confirmed at autopsy for every 16 deceased without emphysema (33/513). A significantly higher number of cases of BCA was found in patients with pulmonary emphysema, $P<0.001$, see Table 3 .

\section{Pleural effusion}

Pleural effusion was identified during life and mentioned in the accompanying reports of the deceased in 93 cases in the studied group (13\%). At autopsy, an effusion was identified in another 140 subjects, for a total of 233 cases out of the 708 deceased (33\%). The effusions were bilateral, right-sided and left-sided in 160, 45 and 28 cases, respectively.

\section{Microscopic examination of the lungs}

Microscopic examination of the lungs was performed by the seventh author of this paper. For this analysis, we examined the samples for the presence of emphysema, chronic bronchitis and pulmonary carcinoma.

In the 610 microscopically examined lungs, emphysema was detected in 330 of the deceased (179 males and 151 females, $54 \%$ ), and focal emphysematous changes were described in another 72 cases ( 37 males and $35 \mathrm{fe}-$ males, 12\%). Thus, findings compatible with pulmonary emphysema were detected in 402 of the deceased (216 males and 186 females, 66\%). Excluding the cases

Table 3. Frequency of lung cancer found in the group of the deceased with and without emphysema.

\begin{tabular}{lccc}
\hline & $\begin{array}{c}\text { Number } \\
\text { of cases }\end{array}$ & $\begin{array}{c}\text { Number of } \\
\text { BCA cases }\end{array}$ & Ratio \\
\hline Whole group & 708 & 55 & $1: 13$ \\
Cases with emphysema & 195 & 22 & $1: 9$ \\
Cases without emphysema & 513 & 33 & $1: 16$ \\
\hline
\end{tabular}

with focal emphysematous changes, microscopically detected emphysema was present in $54 \%$. To clarify the differences between the presence of emphysema detected macroscopically at autopsy and that detected microscopically, we would like to emphasize emphysema was identified during life in only 83 of the deceased (12\%), whereas macroscopic detection identified 195 cases, $28 \%$ of the entire studied set.

Table 4 shows the distribution of microscopic emphysema by age. These data show that when all cases with emphysema including those with only focal emphysematous changes, are considered, the percentage of emphysema increases only slightly with age. In subjects under 40 years of age, emphysema was identified in $59 \%$ of the deceased, in the age group $60-69$ years in $68 \%$ of the deceased and in those over 90 years of age in $74 \%$ of the deceased.

Pearson's chi-square test showed that there was a dependence of the the incidence of microscopic emphysema (excluding the focal changes) on age, the $P$-value of the test was $P=0.022$.

However, an analysis of findings compatible with emphysema (including those with only focal changes), indicated that there was not a statistical correlation between the occurrence of emphysema and age as shown by Pearson's chi-square test; the $P$-value of the test was $P=0.34$.

Evidence for chronic bronchitis was evaluated in 601 lungs. Microscopic signs of chronic bronchitis were detected in 289 cases ( 174 males, 115 females), representing $47 \%$ of the examined subjects.

There were 261 cases in which microscopically proven emphysema coincided with chronic bronchitis. However, there were also 141 cases in which emphysema was detected, but was not accompanied by chronic bronchitis. In contrast, chronic bronchitis without emphysema was found in only 28 of the cases $(10 \%)$.

Microscopic examination to detect lung cancer was performed in 606 of the total set of 708 subjects. In 563 cases, no evidence for BCA was detected. In the remaining 43 subjects, BCA was detected, with adenocarcinoma accounting for 22 of the cases (51\%). Other histological types included squamous carcinoma, small cell carcinoma, undifferentiated carcinoma and carcinoid in 9, 7, 2 and 1 cases, respectively, and in 2 other cases an unspecified histomorphological type was present.

BCA: lung cancer

Table 4. The incidence of microscopically proven emphysema according to age.

\begin{tabular}{lccccccc}
\hline & \multicolumn{7}{c}{ Number of cases (\%) } \\
\hline Age group (years) & $\leq 39$ & $40-49$ & $50-59$ & $60-69$ & $70-79$ & $80-89$ & $\geq 90$ \\
Whole group & 26 & 42 & 68 & 193 & 158 & 186 & 35 \\
Emphysema present & 9 & 10 & 32 & 101 & 80 & 80 & 18 \\
& $(41 \%)$ & $(29 \%)$ & $(56 \%)$ & $(59 \%)$ & $(58 \%)$ & $(51 \%)$ & $58 \%)$ \\
Emphysema incl. focal changes & 13 & 17 & 36 & 116 & 91 & 106 & 23 \\
& $(59 \%)$ & $(49 \%)$ & $(63 \%)$ & $(68 \%)$ & $(66 \%)$ & $(67 \%)$ & $(74 \%)$ \\
Emphysema not present & 9 & 18 & 21 & 54 & 46 & 52 & 8 \\
Not examined & 4 & 7 & 11 & 23 & 21 & 28 & 4 \\
\hline
\end{tabular}


The combination of BCA, microscopically confirmed emphysema and chronic bronchitis was found in 33 of the BCA cases $(76 \%)$. Other BCA cases were accompanied by chronic bronchitis or with emphysema only. Only two subjects with BCA did not have any microscopic evidence for emphysema or chronic bronchitis. See Table 5.

Out of the 208 deceased without microscopically proven emphysema, BCA was found only in 4 of them ( $2 \%)$. In the 402 deceased with microscopic evidence for emphysema, the microscopic analysis identified 39 cases of BCA ( $10 \%$, i.e., almost one in ten of the deceased subjects with emphysema also had BCA).

\section{DISCUSSION}

The goal of this prospectively planned autopsy study was to determine, as accurately as possible, the number of lung conditions, including emphysema, lung cancer, chronic bronchitis and pleural effusion, present in the set of autopsied deceased in the University Hospital, Prague - Motol. The data for this study was been collected over a period of two years, with the goal of including a large study group for witch the results would be minimally affected by errors due to small sample size. We are aware of the limitations of autopsy studies, including a bias toward older age, polymorbidity of the deceased and the diagnostic selection according to the focus of the hospital. The last of these factors should be minimized due to a broad spectrum of care provided for both adults and children by the University Hospital in Motol, Prague.

The number of macroscopically proven cases of emphysema (28\%) in the deceased with a median of 71 years of age significantly exceeds the data of the prevalence of COPD (7.7\%) usually described in our population. This high number may be influenced by the age of the deceased. However, if we take into account microscopically proven emphysema in the group of patients under 40 years of age (emphysema in $41 \%$, focal emphysematous changes in as many as $59 \%$ ) it can be assumed, that the prevalence of emphysema in this population will be higher than expected.

The microscopic detection of emphysema in 54\% (including focal lesions in $66 \%$ ) of the entire group of lungs is comparable to the results presented by the authors of a study performed in Basel, who in 1974 established by autopsy that "destructive pulmonary emphysema" was present in $53.7 \%$ of the 467 deceased $^{5}$. The fact that 39 years ago, emphysema was found 3 times more frequently in males than in females, while in the current group we did not observe degree of difference between males and females, is interesting. In our group, $45 \%$ of the emphysema cases indentified macroscopically at autopsy were females. We were surprised by the fact that emphysema detected during life was more frequent in females than in males (43 vs. 40), whereas at autopsy, this condition was more frequently identified in males than females (67 vs. 45). Another difference between our results and the finding from the study in Basel was the fact that in their group "destructive emphysema" was not found in the deceased
Table 5. Combination of lung cancer, emphysema and chronic bronchitis.

\begin{tabular}{lc}
\hline Microscopically proven diagnosis & Number of cases (\%) \\
\hline BCA + emphysema + chronic & $33(76 \%)$ \\
bronchitis & $6(14 \%)$ \\
BCA + emphysema & $2(5 \%)$ \\
BCA + chronic bronchitis & $2(5 \%)$ \\
BCA only & \\
\hline
\end{tabular}

BCA: lung cancer

who were under the age of 40 years, while we demonstrated the presence of emphysema even in this age group.

The statistical analysis of the dependence of microscopically proven emphysema on age using Pearson's chisquare test showed no statistical dependence.

Similar results were published by Ryder in 1971 (ref. ${ }^{6}$ ). In 353 consecutive autopsies, he detected emphysema in 219 of the deceased (62\%). The extent of emphysema ranged from $0.5-95 \%$ with an average of $12.6 \%$ of the total lung volume being affected. Smoking history was known in 179 cases. Of the known smokers, $75 \%$ had signs of emphysema.

The data presented above indicate that emphysema may be found in more than half of the deceased at autopsy, and currently its prevalence is only slightly greater in males than in females.

The number of lung cancer cases not diagnosed during life $(44 \%)$ was higher than stated in the literature and exceeded our expectations. The results confirm and further define our clinical experience that the incidence of lung cancer among subjects with emphysema is higher (1 out of 9) than in those without emphysema (1 in 16).

According to a similar previous study that was carried out in the Czech Republic, one newly diagnosed lung cancer was detected in every 80 autopsies ${ }^{7}$. In our group we found one "new" BCA case in every 30 autopsies. We may speculate about the number of unrecognized BCA in the Czech Republic. We assumed that all cases of BCA newly identified by autopsy were reported and therefore included in the reported annual incidence of BCA. From official statistics, we know that only approximately $30 \%$ $(31,500)$ of the deceased $(107,000)$ in the Czech Republic were autopsied ${ }^{8}$. We further assume that among the remaining approximately 75,500 of the deceased who were not autopsied, approximately 2500 unrecognized cases of lung cancer during life escaped being submitted to the national oncology register. If we apply our results with the "poor" assumption that all cases of BCA newly identified at autopsy are properly reported and therefore this number contributes to the overall incidence, we obtain the following numbers: the overall incidence of BCA in the Czech Republic is $28 \%$ higher than is officially reported (the absolute number of cases of BCA in 2010 was 6,523 with an incidence of 61.93/100,000) and reached approximately 9,000 , so the real incidence of BCA may be $86 / 100,000$. 
The high frequency of BCA coincident with pulmonary emphysema is obvious from results of lung cancer screening trials. This was also proven in the examination of smokers and ex-smokers using low-dose spiral CT in "The Pittsburgh Lung Screening Study, a sub-project of the University of Pittsburgh Lung Cancer Specialized Program of Research Excellence" (SPORE) (ref. ${ }^{9}$ ). This project studied the incidence of signs of lung cancer and emphysema using chest CT measurements of 3,868 subjects (60\% smokers, $40 \%$ ex-smokers) aged 50-79 years (mean age, 59 years), of whom $49 \%$ were females. During 3 years of repeated CT examinations at one-year intervals the study diagnosed the following numbers of BCA cases: 41 (first year), 43 (second year) and 15 (third year), for a total of 99 BCA cases. Of this number, 32 were also diagnosed with emphysema, bronchitis or asthma. According to the CT results, "any" emphysema (divided into subgroups of "trace", "mild" and "moderate to severe") was detected in 1,546 patients, out of whom 75 had also BCA (21:1). In this screened population, 2,094 participants had no signs of emphysema. In the emphysema-free subgroup, only 24 cases of BCA were detected (87:1). Among the 99 cases of BCA detected by screening, 75 were accompanied by emphysema ( $75 \%)$. The following conditions were proven to be strong determinants for predicting BCA: the existence of obstructive ventilatory disorders $(P<0.0001)$ and emphysema $(P<0.0001)$. The frequency of BCA coincident with emphysema in this CT screening (75\%) was similar to our results; in our study, accompanying macroscopic emphysema was demonstrated in $40 \%$ of subjects with BCA, and microscopic emphysema including focal changes was observed in $90 \%$ of the deceased with BCA.

Similar information can also be obtained from studies in which resected parts of lungs are examined for the coexistence of emphysema with BCA. In one study that focused on CYP1A1 gene polymorphisms in relation to the development of BCA and pulmonary emphysema, 129 lungs were resected due to BCA (ref. $\left.{ }^{10}\right)$. The coexistence of macroscopic emphysema and BCA was detected in $87(67 \%)$ of them, which is a comparable result to our autopsy study. The primary outcome of this study was the proof of the association of the P4501A1 gene polymorphism with an increased risk for the development of emphysema and BCA.

In contrast, a study published in 2012 by other authors found a different proportion of emphysema in patients with BCA (ref. ${ }^{11}$ ). They investigated 100 deceased with advanced lung cancer to determine the cause of death. The most common results included primary tumour progression, infectious complications and metastases to other organs. In this study, they also evaluated the existence of associated diseases that contributed to death in patients with BCA, with emphysema being the most commonly found $(38 \%)$.

Additional results similar to ours were obtained in a study that examined the occurrence and localization of BCA in relation to the extent of emphysema in a group of 153 subjects with biopsy-proven stage I NSCLC. The presence of emphysema was detected in $75 \%$ of these subjects. This study also found the area with the most significant emphysema, the right upper lobe, was also the most common location for BCA $(37 \%, P<0.001)\left(\right.$ ref. $\left.^{12}\right)$.

In 2010 and 2011, Robert Young published several interesting articles on the correlation between BCA and COPD. He reported that smokers with COPD have a 4 to 6 times higher risk of developing BCA compared to smokers without obstruction and that $50-80 \%$ of the subjects diagnosed with BCA are simultaneously diagnosed with COPD, while in smokers without lung cancer, COPD develops only in approximately $15-20 \%$ of cases (ref. ${ }^{13}$ ). This work also broadly discussed a possible common genetic origin of both diseases. A "resistant smoker" is defined as a smoker resistant to developing COPD as well as BCA (approximately 60\%) (ref..$^{14}$ ).

In addition, Japanese authors have been searching for the genetic basis of BCA and emphysema. They showed, that a "single nucleotide polymorphism" (SNP) rs721917, which resulted in the exchange of a methionine for a threonine (the Met11Thr variation), is associated with emphysema, interstitial pneumonia and BCA (ref. ${ }^{15}$ ).

In our set, autopsy-confirmed pleural effusions were present in one third of the deceased, while the accompanying reports of the deceased obtained for the autopsy described an effusion in only $13 \%$. This confirms our clinical experience that this complication is not being considered often enough. In our group, $25 \%$ of those with BCA were also observed to have pleural effusion. According to Prof. Light, pleural effusion is present in $15 \%$ of BCA patients at the time of diagnosis it occurs in $50 \%$ of the patients during the course of the disease ${ }^{16}$.

\section{CONCLUSIONS}

We conclude that our autopsy study will help to refine our estimates of incidence as well as the associations between the observed diseases, especially lung cancer, emphysema and pleural effusions. Despite its limitations, especially the fact that the studied patients died at a mean age of 71 years and were undoubtedly more seriously ill than living people of the same age, we believe that our results will contribute to more thorough clinical examination with regard to an early diagnoses of the disease even in the presence of minimal clinical symptoms. At the same time, we believe that our results will encourage further studies of context and the possible common genetic basis of lung diseases (genetic polymorphisms common to certain diseases), as briefly mentioned above. This information may be a significant help in future diagnoses.

Acknowledgement: This work was supported by Projects (Ministry of Health) of conceptual development of research organization 00064203 (FN Motol, Prague, Czech Republic).

Author contributions: MM, FP, RK, LF: conception and design of the study; HH, LC: acquisition of the data; LK, $\mathrm{ZK}, \mathrm{AG}$ : analysis and interpretation of the data; MM, AG, RK, LF: revision of the manuscript; all authors: drafting and final approval of the version to be submitted.

Conflict of interest statement: None declared. 


\section{REFERENCES}

1. Institute of Health Information and Statistics of the Czech Republic Cancer incidence 2010 in the Czech Republic. Available at http:// www.uzis.cz/publikace/novotvary- 2010

2. Vondra V. The importance of chronic obstructive pulmonary disease Vnitr Lek 2004;50(9):654-8.

3. Bechtel JJ, Kelley WA, Coons TA, Klein MG, Slagel DD, Petty TL. Lung cancer detection in patients with airflow obstruction identified in primary care outpatient practice. Chest 2005;127(4):1140-5.

4. Marel M, Zrustova M, Stastny B, Light RW. The incidence of pleural effusion in a well-defined region. Epidemiologic study in central Bohemia. Chest 1993;104(5):1486-9.

5. Dalquen P. Incidence of pulmonary emphysema, a study of 467 randomized autopsy cases. Beitr Pathol 1974;153(4):330-8.

6. Ryder RC, Dunnill MS, Anderson JA. A quantitative study of bronchial mucous gland volume, emphysema and smoking in a necropsy population. J Pathol 1971;104(1):59-71.

7. Ostadal O. Neglected point of view in the epidemiology of broncho genic carcinoma. Stud Pneumol Phthiseol 1994;54(1):28-30.

8. Institute of Health Information and Statistics of the Czech Republic Activity of common examination and treatment units 2010 . Available at http://www.uzis.cz/book/export/html/4623.

9. Wilson DO, Weissfeld JL, Balkan A, Schragin JG, Fuhrman CR, Fisher SN, Wilson J, Leader JK, Siegfried JM, Shapiro SD, Sciurba FC.
Association of radiographic emphysema and airflow obstruction with lung cancer. Am J Respir Crit Care Med 2008;178(7):738-44.

10. Cantlay AM, Lamb D, Gillooly M, Norrman J, Morrison D, Smith CA, Harrison DJ. Association between the CYP1A1 gene polymorphism and susceptibility to emphysema and lung cancer. Clin Mol Pathol 1995;48(4):M210-4.

11. Nichols L, Saunders R, Knollmann FD. Causes of death of patients with lung cancer. Arch Pathol Lab Med 2012;136(12):1552-7.

12. Bishawi M, Moore W, Bilfinger T. Severity of emphysema predicts location of lung cancer and 5-y survival of patients with stage I nonsmall cell lung cancer. J Surg Res 2013;184(1):1-5.

13. Young RP, Hopkins RJ, Christmas T, Black PN, Metcalf P, Gamble GD. COPD prevalence is increased in lung cancer, independent of age, sex and smoking history. Eur Respir J 2009;34(2):380-6.

14. Young RP, Hopkins RJ, Gamble GD, Etzel C, El-Zein R, Crapo JD. Genetic evidence linking lung cancer and COPD: a new perspective. Appl Clin Genet 2011:4:99-111.

15. Ishii T, Hagiwara K, Ikeda S, Arai T, Mieno MN, Kumasaka T, Muramatsu M, Sawabe M, Gemma A, Kida K. Association between genetic variations in surfactant protein $d$ and emphysema, interstitial pneumonia, and lung cancer in a Japanese population. COPD 2012;9(4):409-16.

16. Light RW. Pleural effusions related to metastatic malignancies. In Light RW. Pleural diseases, $4^{\text {th }}$ ed. Lippincott Williams \& Wilkins. Philadelphia, PA, USA 2001: p.108-134. 